\title{
Analogues in thermodynamics: the Partial Derivative Machine and Legendre transformations
}

\author{
Michael Vignal, ${ }^{1}$ Corinne A. Manogue, ${ }^{1}$ David Roundy, ${ }^{1}$ and Elizabeth Gire ${ }^{1}$ \\ ${ }^{1}$ Department of Physics, Oregon State University, Corvallis, OR 97331
}

\begin{abstract}
One commonly and persistently difficult topic in thermodynamics is the relationship between the different thermodynamic potentials obtained through Legendre transformations. The Partial Derivative Machine (PDM) was developed at Oregon State University to be a mechanical analogue of a thermodynamic system. This analogue allows students to explore the mathematics of thermodynamics, including Legendre transformations, on a mechanical system that students can understand without having to first learn new physical concepts. We conducted 12 teaching interviews with middle-division undergraduate physics majors to explore student usage of the PDM. In these interviews, we taught interview participants Legendre transformations on the PDM and then asked them to perform a thermodynamics transfer problem. We found that participants used the PDM productively and in different ways while performing the transfer problem. Furthermore, many participants stated that the teaching interview helped their understanding of Legendre transformations.
\end{abstract}

\section{INTRODUCTION}

Manipulatives can help students explore and better understand physical phenomena [1]. Physical manipulatives (as opposed to virtual manipulatives) can help students develop "a sophisticated epistemology of science" that spans both content and experimentation. In an effort to foster student learning in thermodynamics, the Oregon State University Physics Education Research Group developed the Partial Derivative Machine (PDM) as a mechanical analogue to a thermodynamic system [2]. The PDM (Fig. 1) allows students to explore challenging aspects of thermodynamics, including inaccessible variables and thermodynamic potentials, on a mechanical system that students can understand without having to first learn new thermodynamic concepts [3]. We conducted post-instruction teaching interviews [4] with 12 undergraduate physics majors who had used the PDM multiple times in a junior year thermodynamics unit. Through these interviews, we attempted to learn about student understanding and use of the PDM by exploring the following research questions:

RQ1 Do students understand the PDM as a mechanical device?

RQ2 Do students understand the PDM as an analogy for a thermodynamic system?

RQ3 Do students transfer their understanding from the PDM to thermodynamics systems?

To explore these questions, we selected the topic of Legendre transformations for our interview. In thermodynamics, Legendre transformations are used to derive thermodynamic potentials such as enthalpy (Fig. 2) and the Gibbs free energy. However, Legendre transformations are typically under-emphasized in classroom instruction and poorly understood by students [5]. Our interview participants had spent only a part of one 50minute class period learning about Legendre transformations (done without reference to the PDM). In our interviews, we taught participants how to perform Legen-

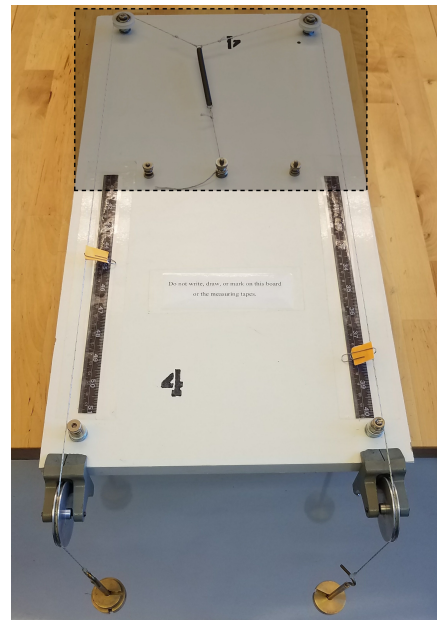

FIG. 1. A Partial Derivative Machine (PDM) with a representation of a black box that can be physically placed on the PDM to hide the system. Two strings emerge from the box/system, each with an associated position (indicated by flag markers) and force (provided by hanging masses).

dre transformations on the PDM and discussed why they are useful. We then gave them a thermodynamic transfer problem that required them to motivate, choose, and perform a Legendre transformation.

\section{METHODS}

We collected interview data from 12 middle-division undergraduate physics majors at a large four-year public research-intensive university across 2 academic years. Guided by a phenomenographic framework, we sought to understand the different ways that students use and understand the PDM [6]. While student experiences with the PDM in class and in the interview differed between years, these differences provided participants with a wider range of experiences that in turn enriched our phenomenographic study.

We used a progressive refinement technique [7] to develop $\sim 50$ minute-long semi-structured teaching inter- 


$$
\begin{array}{rlrl}
\underline{\mathrm{PDM}} & \text { Thermodynamics } \\
d U & =F_{1} d x_{1}+F_{2} d x_{2} & d U & =T d S-p d V \\
d A & =\_d x_{1}+\ldots d F_{2} & d H & =\_d S+\_d p \\
A & =U-F_{2} x_{2} & H & =U+p V \\
d A & =d U-F_{2} d x_{2}-x_{2} d F_{2} & d H & =d U+p d V+V d p \\
d A & =F_{1} d x_{1}+F_{2} d x_{2} & d H & =T d S-p d V \\
-F_{2} d x_{2}-x_{2} d F_{2} & & +p d V+V d p \\
d A & =F_{1} d x_{1}-x_{2} d F_{2} & d H & =T d S+V d p
\end{array}
$$

FIG. 2. Derivation of the differential enthalpy $d H$ and its PDM analogue $d A$ through a Legendre transformation. Starting from the first law of thermodynamics (1), we wish to obtain a target equation (2) with specific independent variables. (3) is the Legendre transformation that defines enthalpy $(H$ or $A$ ). We then 'zap with d' (4), substitute (1) into (4) to yield (5), and then simplify to find the target equation (6).

views. Participants were provided a PDM and large whiteboards and asked to articulate their thinking and reasoning aloud. Author MV pilot tested the interview protocol 3 times, twice with graduate students and once with author DR. We made revisions after each pilot interview. MV then interviewed 5 students 2-4 weeks after their thermodynamics unit had ended. After a preliminary open coding [8], we made minor adjustments to the classroom presentation of the PDM (introducing cycles on the PDM) as well as to our interview protocol (interview organization and word choice). MV then interviewed 7 students with this modified protocol during the following academic year (10-12 weeks post instruction), for a total of 12 interviews. This final protocol is summarized below:

1. Legendre transformation recall questions:

(a) What is a Legendre transformation?

(b) Prompt: What about the Gibbs free energy?

2. PDM recall questions

(a) What is the PDM?

(b) Prompt: What can you measure on the PDM?

(c) What can you say about how the PDM relates to thermodynamics?

3. Teaching Legendre transformations on the PDM

4. Thermodynamic Legendre transformation transfer problem (Fig. 3)

5. Reflection

(a) What did you think of this interview?

(b) Did the PDM help you solve the transfer problem? If so, in what way(s) did the PDM help?

Legendre transformation and PDM recall: In the recall portions of the interview, MV asked participants what they knew or remembered about Legendre transformations and the PDM. This would later help contextualize each participant's performance on the transfer problem. Further prompting, such as asking explicitly about the Gibbs free energy or what one can measure

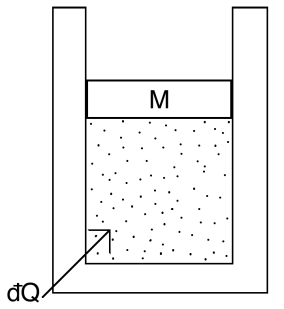

Consider a gas in a chamber in equilibrium with a massive piston (free to slide up and down) on top. Suppose we add an amount of heat $₫ Q=T d S$ to the gas (the system is otherwise thermally isolated). A change in which thermodynamic potential would be the easiest for us to measure?

FIG. 3. Interview transfer problem and provided diagram.

with the PDM, was provided to participants who indicated familiarity with these topics but who demonstrated no further understanding. MV also asked the participants what they could say about why the PDM was used in the thermodynamics unit in particular.

Teaching: In the teaching portion of the interview ( $\sim 25$ minutes), MV walked participants through Legendre transformations on the PDM and encouraged the participant to ask questions and seek clarification. The left column of Fig. 2 contains the equations used in this portion of the interview.

Participants were first asked to consider equation (1), $d U=F_{1} d x_{1}+F_{2} d x_{2}$, where $U$ is the internal energy of the system and the $F$ s and $x$ s represent the forces and positions on either side of the PDM. The PDM's system is considered to be whatever is under the black box, represented as the shaded rectangle in Fig. 1. The terms $F_{1} d x_{1}$ and $F_{2} d x_{2}$ in $d U$ represent infinitesimal amounts of work that could be done on either side of the PDM to change its internal energy. MV pointed out how this equation is analogous to the first law of thermodynamics, $d U=T d S-p d V$ (where $T, S, p$, and $V$ represent temperature, entropy, pressure, and volume respectively), but from this point on he discussed the PDM only as a mechanical system and not as an analogy. MV discussed how $x_{1}$ and $x_{2}$ were the independent variables in $d U$ and noted that $d U$ would be useless if $x_{2}$ was, for some reason, impossible to measure. If, however, we knew that the mass on the hanger did not change, then $F_{2}$ would become an independent variable. This motivates the target equation (2) in which $x_{1}$ and $F_{2}$ are the independent variables. MV then described a Legendre transformation ( $\left.A=U-F_{2} x_{2}\right)$, which was 'zapped with d' [9] to obtain a differential form of this transformation $(d A)$ that had the appropriate independent variables (6). MV also pointed out that, in this particular case, $d F_{2}$ was zero since $F_{2}$ was constant, and thus $d A=F_{1} d x_{1}$. MV and the participant then discussed this enthalpy-like quantity by considering work and conservation of energy in order to obtain a physical understanding of $A$ and $d A$.

Transfer: During the thermodynamics transfer problem, we provided participants the prompt shown in Fig. 3. To obtain an appropriate thermodynamic potential for this system (a gas-filled piston to which we add heat), one must first identify the system's independent variables (heat $Q$ and pressure $p$ ). This motivates a Legendre transformation (Fig. 2, right column) to obtain the thermodynamic potential enthalpy, which has those inde- 
pendent variables. We phrased the question in terms of identifying the easiest thermodynamic potential to measure in the hope that participants would recognize that heat is given and pressure is constant. This problem is analogous to the situation in the teaching portion of the interview (as can be seen by the parallels in the equations in Fig. 2) when the mass providing $F_{2}$ is kept constant.

We transcribed all 12 interview recordings in full and imported them into Dedoose, a qualitative analysis program, for a more thorough open coding.

\section{RESULTS}

We found no distinct patterns in our coded analysis that differentiated participants in one academic year from participants in the other. This suggests that individual background knowledge and experiences, rather than differences in instruction, contributed to the majority of differences that we saw between participants.

Legendre transformation recall: Participants' initial recall of Legendre transformation was sparse. Three of the 12 participants explicitly stated that they do not believe Legendre transformations were taught in class. Only 1 participant correctly performed and explained a Legendre transformation at this point in the interview, and 3 others wrote down an equation that was incorrect but resembled a Legendre transformation.

PDM recall: Participants' initial recall of the PDM as a mechanical device (RQ1) was strong. All participants indicated familiarity with the PDM, demonstrating understanding of the variables that could be measured (2 positions and 2 forces), and that what lay under the black box related these 4 variables. Ten of the 12 participants discussed measuring these relations (e.g. through partial derivatives) on the PDM.

Sam We looked at like every variable that you could control, like the mass [gestures at right mass], where your starting distance was [gestures at right position marker], whether or not you are holding [the right position] constant so it would not be able to move... . And then how changing 1 of those variables affects the other variables in the system.

Participants' initial understanding of the PDM as a thermodynamic analogy (RQ2) was mixed. We identified three areas in which participants indicated understanding of this analogy: the PDM can model a state system $(n=4)$; the the PDM can simulate the inaccessibility of the system or of particular variables $(n=6)$; and the PDM can be used to find relations between different variables in a way that related to thermodynamics $(n=9)$. These different understanding are demonstrated in the following 2 quotes:

Gabriel We also used [the PDM] to demonstrate that you can describe a certain state of a system using a minimum number of variables.
Like, in here [gestures at black box] there was the spring-strings coming off the 2 different sides, and you could describe [the system] based on I think just 2 variables.

Jesse [The PDM is] an analogy for a system where we can measure things, you know, things that are changing. We change this, how much does this other property change, but we don't know exactly what's going on in the system because, in [thermodynamic] systems, we often can't know what's going on in the system because it's all molecular, atomic. Billions and billions of parts.

While every participant offered at least 1 of these 3 descriptions of the analogy, only 1 participant offered all 3 .

Transfer: The participants' use of the PDM to aid with the transfer problem (RQ3) was also mixed. Nine participants explicitly referred back to the PDM while solving the transfer problem, and 1 other participant later claimed to have referenced the PDM while solving the transfer problem but without making this reference explicit in the moment. Of these 10 participants, half referred back to the PDM with no prompting and half did so with prompting. Nine of the 10 participants who referred back to the PDM referenced equations or expressions to help them solve the transfer problem.

Alex I am going to use your [gestures at PDM equations] route of thinking here. We're going to start with $U$. . but now we want things in terms of $d p$ instead of $d V$, so we want to swap $d V$ and $d p$, so we actually want to add $P V$, which $I$ believe is $H$ [writes $U+P V=$ $H]$.

Kai If pressure's gonna be constant... before we were considering the $x s$, but we wanted to talk about $F_{2}$, so I think we want to get to the point where we can talk about a dp.... So we could do a LT where we're gonna... add a VP to, I want to say, U. Right? [writes $d A=U+V P]$.

Only 4 participants referred back to the physical machine itself, with only 2 of them actually manipulating the PDM while working on the transfer problem.

Reflection: Ten participants claimed that the PDM helped them complete the transfer problem, including 1 participant for whom we have no evidence of them referring to the PDM during the transfer problem.

Participants claimed that they benefited from using the PDM in various waves. They expressed that the PDM was helpful: to touch and manipulate $(n=2)$; as a visual reminder to cue the analogy of the PDM $(n=6)$; as a physical/mechanical device that was easy to understand (eg. it was easy to see what was inside and outside the system) $(n=5)$; and because the equations for the PDM and a thermodynamic system are similar $(n=6)$. 
MV How much do you think talking about [the $\mathrm{PDM}$ ] helped you do this [transfer] problem?

Elliott I mean, it definitely helped me understand where [the thermodynamic potentials] came from. Cuz doing it in class, it was kind of just like, 'Here is these things, have fun, bye!'... At least doing the math for this part [gestures at equations for PDM] definitely helped me figure out how to do the math for just this [gestures at equations for transfer problem], and to determine the equations for $d A \ldots$ instead of just like pulling them out from somewhere.

$\boldsymbol{M V}$ Do you think this would have been as effective if [the $\mathrm{PDM}$ ] was not actually sitting here?

Elliott I like to like touch things when I learn. I don't necessarily even have to do, like, the hands on part, but just the fact that I can see [the PDM] and I don't have to do the extra work to like imagine it was like nice. Especially cuz you can actually feel [pulls on right string] that you have to change the work [sic] ... if you do a large change.

All 6 participants who said that the equations were helpful had explicitly referenced them in the transfer portion, but the 2 who said that having the PDM to touch and manipulate was helpful did not actually do so while solving the transfer problem (and the 2 who did touch the PDM during the transfer problem did not cite being able to touch the PDM as helpful in the reflection). Further study is needed to better understand connections and discrepancies between what students know and say about the PDM and how they actually use it.

\section{IMPLICATIONS FOR INSTRUCTION AND CONCLUSIONS}

Initial student understanding of the PDM as a mechanical device was strong, but understanding of it as an analogy for a thermodynamic system and student transfer ability were mixed.

All 12 participants eventually successfully completed the thermodynamics transfer problem, even those who originally stated that they never learned Legendre transformations in class. This suggests learning about Legendre transformations on the PDM was useful for the participants. Furthermore, many participants explicitly claimed that they found this experience useful. Eight participants stated that they understood Legendre transformations and thermodynamic potentials better at the end of the interview than they ever did in the course. Specifically, participants' claimed that their learning was fostered by: having the interview as a refresher on Legendre transformations $(n=4)$; engaging in a one-on-one learning experience $(n=2)$; and having such a strong focus on independent variables to motivate Legendre transformations $(n=8)$. These claims suggest positive student affect, which we believe is an important asset of the PDM.

\section{Sam When you teach the next people thermody- namics, doing something like this and hav- ing that discussion would be extremely helpful. [Thermodynamic potentials] just like turned up one day, and then we just kept going.}

We acknowledge that the interview participants might have experienced real or perceived social pressure to respond positively when asked about the interview by the interviewer. However, we do have evidence that some of the participants recommended participating in the interview to others, which suggests that at least some of the participants genuinely found the experience worthwhile.

Parker I was really excited to do this [interview]. I heard from a couple of people that it wasthey didn't tell me anything about it, they just said 'yeah, you want to do it!'

Further research might look at groups of students using the PDM, which is how the PDM is used in class. Such research could inform adaption of our interview protocol for teaching Legendre transformations in the classroom.

\section{ACKNOWLEDGEMENTS}

We would like to acknowledge the Oregon State University Physics Education Research Group. This research was supported in part by NSF DUE-1323800.
[1] T. De Jong, M. C. Linn, and Z. C. Zacharia, Science 340, 305 (2013).

[2] G. Sherer, M. B. Kustusch, C. A. Manogue, and D. Roundy, in PERC Proceedings (2013) pp. 341-344.

[3] D. Roundy, M. Bridget Kustusch, and C. Manogue, Am. J. Phys. 82, 39 (2014).

[4] J. Kilpatrick, in Proceedings of the international group for the psychology of mathematics education. Volume 1 (University of Montreal, 1987) pp. 3-27.

[5] R. K. Zia, E. F. Redish, and S. R. McKay, Am. J. Phys. 77, 614 (2009).
[6] F. Ornek, in Asia-Pacific Forum on Science learning and teaching, Vol. 9 (2008) pp. 1-14.

[7] R. A. Engle, F. R. Conant, and J. G. Greeno, Video research in the learning sciences , 239 (2007).

[8] M. Miles, A. Huberman, and J. Saldaña, Qualitative Data Analysis (SAGE Publications, 2013).

[9] T. Dray and C. A. Manogue, The College Mathematics Journal 41, 90 (2010). 